\title{
Recall of Events Affects Perception of Happiness
}

\author{
Angelica Moè \\ University of Padua, Padua, Italy
}

\begin{abstract}
This study assessed the relationships between recall of positive and negative events, API (attention to positive) and ANI (attention to negative information), and perception of happiness, with the hypothesis that recall affects the perception of happiness and that ANI and API affect recall. One hundred and five women filled in the APNI scale to assess API and ANI and were asked to listen to and recall a story presenting both positive and negative events, and provide an assessment of the character perceived happiness. Finally, they were asked to choose an ending for the story. Results showed that recall is related to the perception of happiness more than API and ANI, and that relationships occur among recall, API, ANI, and perception of happiness. A positive ending for the story was preferred. Discussion focuses on the implications of individual differences in paying attention to and recall positive and negative information.
\end{abstract}

Keywords: recall, happiness, individual differences

\section{Life Is Not What One Lived, But What One Remembers and How One Remembers It in Order to Recount It}

\section{Gabriel Garcia Márquez}

Research has long studied ways for improving recall with the aim to favour learning (e.g., De Beni \& Moè, 2003; Moè \& De Beni, 2005). However, recall is important not only for educational purposes. What we recall can affect our happiness. Recalling positive events, joyful experiences, and cheerful people induces happy judgments, while retrieving bad events, frustrating experiences, sad people, and negative facts induces unhappy ones. This is the leading hypothesis of this study.

Diener and Biswas-Diener (2008) demonstrated the validity of a technique called AIM (Attention, Interpretation and Memory) based on paying attention, doing interpretations in a positive way and recalling positive events or the positive side of neutral or even negative events. They also introduced the "savouring" construct which means staying on recall of the positive in order to increase happiness.

Here, the assumption that happiness could depend on the content of recall was tested experimentally, by examining the link between recall of positive and negative events and perception of happiness. To the best of our knowledge, this has never been explored before, while previous studies have found that a stable tendency in focusing more on positive or negative information affects perception of happiness (Noguchi, Gohm, \& Dalsky, 2006).

This individual difference in attending to the positive or negative aspects of events which occur to

\footnotetext{
*Acknowledgements: The author would like to thank Roberto Micciulla, Marta Romanato, Giorgia Roncon, and Francesca Tessari for their help in data collection.

Angelica Moè, Department of General Psychology, University of Padua, e-mail: angelica.moe@unipd.it.
} 
ourselves and to others is called API (attention to positive information) and ANI (attention to negative information). Research has shown that it has an influence on people's lives, because it can lead them to assess events which happen to them or others in a more positive or negative manner (Dalsky, Gohm, Noguchi, $\&$ Shiomura, 2008) and to recollect positive and/or negative aspects of events in the long term (Noguchi, Gohm, Dalsky, \& Sakaamoto, 2007). More interestingly, Noguchi et al. (2006), studying the relationships of API and ANI with the perception of happiness of a character in a story read aloud, showed that the more people attend to positive events, the higher their evaluation of happiness, while ANI did not relate with perception of happiness.

This tendency in focusing on positive (API) or negative (ANI) events can be measured with a questionnaire devised by Noguchi et al. (2006). They demonstrated that the two dimensions, API and ANI, are differently related to affective, dispositional, and motivational factors. API is positively related to positive effect, optimism, life satisfaction, reward sensitivity, fun seeking, affiliation, extraversion, agreeableness, conscientiousness, openness to experience and negatively related to negative effect and neuroticism. ANI is positively related to negative effect, behavioural inhibition system, neuroticism, and negatively related to life satisfaction, and optimism.

However, the heuristic value of API and ANI in predicting other cognitive functions such as recall has never been examined. This is surprising, because of the central role played by recall in shaping judgments, such as those relating to the perception of happiness (Kemp, Burt, \& Furneaux, 2008) and in leading us to pay attention to congruent information (Bower, 1981).

It therefore appeared interesting to test the hypothesis that happiness judgments are related not only to API and ANI but also to the number of positive and negative events recalled, which may differ among participants because of their different life histories. Hence, to control the ratio of positive to negative events, rather than asking participants to judge their own lives, in this research we used the same story, read aloud, for all our subjects, which narrate events happening to a same gender character with whom participants can identify. It must be noted that judging a character's happiness is not the same as judging one's own happiness. However, this methodological device greatly increases control over the quantity/quality of events on which a person focuses when recalling. Having asked subjects to recall personal life histories should have confused the effects due to the percentage of positive over negative events (different for each participant) with those due to their recall.

It was predicted that greater recall of positive events would correspond to higher perception of happiness and that greater recall of negative events would lower it. API and ANI were also expected to be related with perception of happiness, increasing or decreasing it. Noguchi et al. (2006) found that API but not ANI relates with perception of happiness. This result may depend on the story used, which contained the same number of positive and negative events and may thus persuade participants to rate the character as moderately happy. Here a story having mainly negative events was used in order to induce participants to provide unhappy judgments. This in turn would favor the occurrence of relationships with API, ANI, and recall. In addition here, perception of happiness was measured along a continuum rather than a "yes" or "no" question.

A further aim was to test the mediation of recall. API and ANI may affect perception of happiness directly or through the mediation of the recall of positive or negative events. It was hypothesized that paying attention would favour the recall of either positive or negative events and, in turn, that recall would influence the 
happiness judgment. Lastly, predictions were made about the endings chosen. Participants were presented four endings (two positive and two negative) and asked to choose one. We expected that recall of positive events and API would lead more participants to choose positive endings and that recall of negative events and ANI would lead them to do the opposite.

\section{Method}

\section{Participants}

A convenience sample of 105 women participated on a voluntary basis. Seventy-five were college students, enrolled for course requirement, and 30 were workers. Their mean age was 26.08 ( $S D=9.82)$, age range 19-57.

\section{Materials}

Attention to positive (API) and negative (ANI) information was assessed on APNI (the attention to positive and negative information scale), a self-assessment instrument composed of 22 items: 11 assess API (e.g., "I pay attention to things that lift me up") and 11 assess ANI (e.g., "I can't forget the times I have performed poorly at something"). Participants were asked to rate them on a 5-point Likert-type scale ranging from 1 ("Very untrue of me") to 5 ("Very true of me"). The instrument devised by Noguchi et al. (2006) was validated in Italian (deleted for peer review) and gave the following values: $\chi^{2} / d f=3.28$, RMSEA $=0.07$, GFI $=$ 0.87 , AGFI $=0.85$, SEMR $=0.08$, satisfactory, according to Hu and Bentler (1999). Here, the alpha values for API and ANI were 0.73 and 0.66 , respectively, close to those obtained in the English validation.

A story about a young woman experiencing many problems, such as difficulty at school, divorced parents, being involved in an accident, and being robbed, containing seven negative events and three positive events (201 words) was devised and tested in a small sample of college students $(n=33$, aged 20 to $35, M=22.94, S D$ $=2.70,8$ men) participating for extra credit. They were asked to rate each event on a 7-point Likert scale from "Absolutely negative" to "Absolutely positive", "4" being the mid-point ("Neither negative nor positive") and to recall the story. The mean value for negative events (nos. 1, 3, 4, 5, 6, 9 and 10) was 2.41, $S D=0.50$ (means for each evaluation from 1.97 to 2.85; see Appendix A) and that for the positive events was 5.90, $S D=0.49$ (means from 5.76 to 6.15 ). The difference between the two was significant, $t(32)=31.98, p<0.001$, thus indicating that both positive and negative events are perceived as such. Finally, four alternative endings to the story were prepared. Stemming from the model of Russell (1980), endings (a) and (d) expressed positive effect with high and low arousal, respectively, and endings (b) and (c) expressed negative effect with low and high arousal, respectively (see Appendix B for the four endings).

\section{Procedure}

Participants, tested in small groups, were asked to fill in the APNI scale, without time limits. Then they listened to the story, recorded by a female voice. This task was presented with the following instruction: "Please listen to a short passage lasting 90 seconds". After, they were given a sheet of paper and asked to write down all the information they could remember, without time limits. Then, they were asked to express their perception of the happiness of the character of the story by putting a cross on a 10-cm line ranging from "Very unhappy" to "Very happy". Finally, they were required to choose an ending for the story among the four presented (see Materials). The instruction was "In your opinion how will the story end? (chose one of the following options by putting a cross on (a), (b), (c), or (d))". 


\section{Results}

\section{Scoring}

To obtain the API score, the following items were summed and then divided by $11: 1,6,7,9,11,13,16$, 17, 18 and 20. To obtain the ANI score, the following items were summed and then divided by $11: 2,3,4,5,8$, $10,12,15,19,21$ and 22 . The number of positive and negative events which participants recalled was then computed by the two researchers who collected the data. One point was given for each event correctly mentioned. The agreement was $91 \%$ and the few doubtful responses (e.g., events recalled incompletely) were resolved by a third judge (the first author) and a score of " 0 " or " 1 " was assigned, according to the closeness of the recall to the meaning of the event. The final score was given by the sum of the number of recalled events. Perception of happiness was measured according to the point on the line at which the cross was placed (to two decimal places).

\section{Relationships Among Variables}

A Pearson correlation was run among API, ANI, recall of positive events, recall of negative events, and perception of happiness. As age was related with recall $(r=-0.40, p<0.001)$, it was partialled out. Table 1 lists the results, together with descriptive statistics. Participants recalled the whole story adequately: mean recall of positive events was 2.25 out of a maximum of $3(75 \%)$, and that of negative events 5.82 out of a maximum of 7 (83\%). API and ANI were not related. As expected, owing to the content of the story, participants rated the character as rather unhappy (3.73 out of a maximum of 10). Perception of happiness correlated with recall of positive $(r=0.28, p=0.004)$ and negative $(r=-0.21, p=0.031)$ events and marginally with ANI $(r=-0.18, p=$ 0.077). ANI correlated marginally with recall of negative events $(r=0.19, p=0.051)$ and API with recall of positive events $(r=0.19, p=0.056)$.

Table 1

Correlations Among Variables and Descriptive Statistics ( $n=105)$, Age Being Partialled out

\begin{tabular}{llllllll}
\hline Variables & $M$ & $S D$ & 1 & 2 & 3 & 4 & 5 \\
\hline 1. API (1-5) & 3.74 & 0.38 & --- & & & & \\
2. ANI (1-5) & 3.87 & 0.41 & 0.06 & --- & & & \\
3. Recall of positive events (0-3) & 2.25 & 0.74 & $0.19^{\dagger}$ & 0.04 & --- & & \\
4. Recall of negative events (0-7) & 5.82 & 1.19 & 0.15 & $0.19^{\dagger}$ & 0.17 & --- & \\
5. Perception of happiness (0-10) & 3.73 & 1.43 & 0.15 & $-0.18^{\dagger}$ & $0.28^{* *}$ & $-0.21^{*}$ & -- \\
\hline
\end{tabular}

Notes. ${ }^{*} p<0.05,{ }^{* *} p<0.01,{ }^{\dagger} p<0.10$.

\section{The Mediation of Recall}

Two separate regression analyses were then run, one for API and recall of positive events, and the other for ANI and recall of negative events. Lastly, mediation of recall was tested in both analyses.

As Figure 1 shows, ANI affected perception of happiness through the mediation of recall of negative events. The direct relationship between ANI and perception of happiness was only marginally significant, and disappeared when the mediation of recall of negative events was considered. Similarly, API affected perception of happiness through the mediation of recall of positive events, and the direct relationship became ns when affect of recall was taken into account.

Although the story to which participants listened was quite sad, more attention to positive events and less 
attention to negative events corresponded to higher perception of happiness. Recall of positive and negative events mediated the relationship. This confirms the mediation of recall in the relationship between ANI/API and perception of happiness.

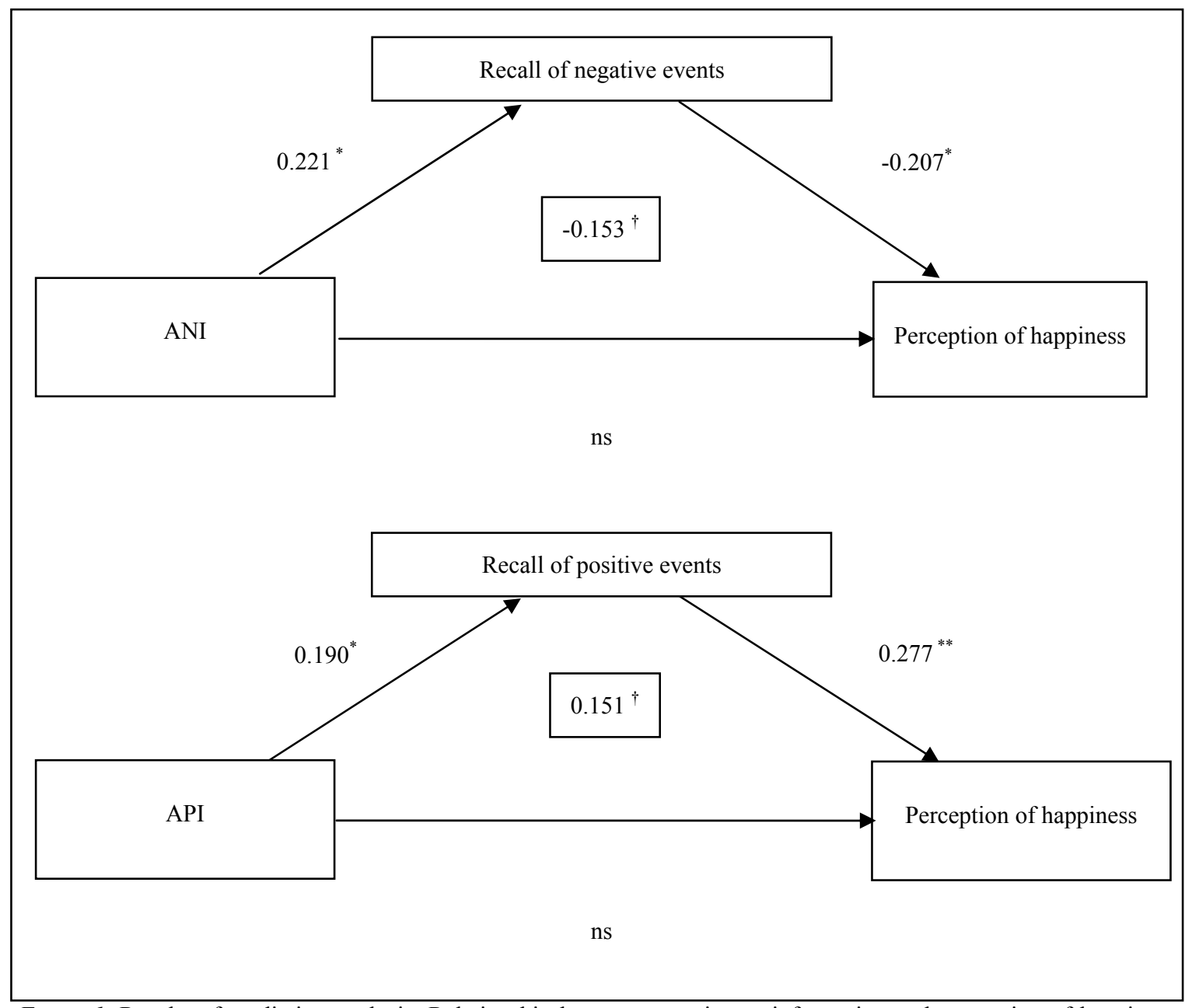

Figure 1. Results of mediation analysis. Relationship between attention to information and perception of happiness becomes not significant when recall of events was controlled for. Arrows top: direct relationship, bottom: mediated relationship. Notes: Figures are beta values, ${ }^{* *} p<0.01,{ }^{*} p<0.05,{ }^{\dagger} p<0.10$, mono-dyrectional hypothesis.

\section{Preferred Ending for the Story}

The choice of story ending was then examined. Overall, $57 \%$ and $35 \%$ of participants chose positive endings (a) and (d), respectively. Only $4 \%$ and $4 \%$ chose negative endings (b) and (c). This means that no matter how much positive and negative information participants recalled, or if they had an high or low API and ANI score, a large majority corresponding to $92 \%$ chosen a positive ending for the story. These results suggest a regulatory process (e.g., Martin \& Clore, 2001), rather than a congruency affect: The character is expected to improve her life.

It should be noted that the positive endings are mainly character-dependent and negative endings are more luck-dependent. Participants may prefer character-dependent to luck dependent endings. In order to examine this possibility, new negative character-dependent endings were devised and tested (see Appendix B points (b)1 and (c)1). "An aunt, to whom Mary is very attached, dies suddenly. Mary is very upset. She thinks there is 
nothing else worth doing and is very sad", was replaced with "Mary quarrels with her boy-friend, they split up, and she falls into a state of great sadness. She thinks it will be impossible to get back in touch with him and is upset", and "Mary witnesses a car accident in which the driver is badly hurt. She is so upset that she cannot do anything. She trembles and cannot even manage to call for help" with "Mary cannot overcome all the misunderstandings among the members of the band. Although she likes playing, she decides to withdraw. She is angry with herself and feels sorry about the decision which has been taken". These new endings were proposed to a group of 33 participants different from those of the study, who were also asked for positive or negative evaluations of each event. All participants chose the positive endings: (a) $76 \%$ and (d) $24 \%$. The regulatory process was thus confirmed also with the new endings.

\section{Discussion}

The results confirmed the hypothesized relationship of recall with perception of happiness. Even if the character underwent many negative events, greater recall of the few positive events corresponded to higher perception of happiness. This adds information to the existing literature (e.g., Emmons \& McCollough, 2003), showing that thinking about positive events favors physical and psychological well-being. API and ANI affected recall of positive and negative events respectively, which in turn affected perception of happiness.

It was difficult to rate high perception of happiness for a character whose misadventures were more numerous than positive events. However, $17 \%$ of participants scored higher than the mid-point of 5 . Low ANI and poor recall of negative events and high API and good recall of positive events helped this high evaluation of happiness.

In the main, a positive ending for the story was chosen, even if the character underwent mainly negative events. This result suggests that participants prefer a positive ending in any case, and are not affected by the quantity of positive and negative events recalled or API/ANI.

Noguchi et al. (2006) found relationships only with API, not ANI. However, the present research differs from that of the above authors because perception of happiness was assessed by means of a single continuum measure rather than a "yes/no" question and participants were asked to choose an ending for the story. That the negative (recall of negative events and ANI) mattered may be interpreted in the light of the negativity bias, that is the general greater tendency to recall and focus on negative rather than positive information (Rozin \& Royzman, 2001).

It has been found that what is recalled can help in providing better evaluations of happiness, especially when positive events are retrieved in the face of misadventures. In addition, individual differences in ANI have been shown to affect the recollection of negative events and, consequently, the assessment of happiness. Future research should explore ways for reducing ANI and sustain recall of positive events, considering longitudinal rather than cross-sectional studies.

In addition, it would be interesting to assess long-term effects. Our results were obtained in only one experimental session, and we therefore cannot speculate on the more durable effects of a chronic tendency to pay more attention to negative as opposed to positive information. A second point relates to the inference that judging the character in a story as happy or unhappy may represent transferral of the same evaluation in assessing one's own life. Some mechanisms will probably differ. However, using a story having as its main character a person with whom our participants could identify seemed to us a way of avoiding confounding effects due to the story itself (a happy/unhappy life) with those due to recall and/or attention to positive and 
negative information. In this research, only female participants and characters were considered. In order to generalize the results properly, future studies should also include male participants and male characters. Finally, the results are based on just a single story. In future studies, it would be interesting to extend the results here obtained with different stories and type of events.

\section{Conclusions}

Being happy and satisfied with life are facets of well-being which favor psychological adjustment, physical health, resiliency in the face of adversity, and longevity (e.g., see, for a review, Diener \& Biswas-Diener, 2008). A wide range of factors can help in this process. The present research stresses the importance of recall and attention to positive and negative information. Our results confirm that individual differences in attending to and recalling events affects perception of happiness. Remembered life is not "lived" life, but it is one of the aspects which can help us toward better living.

\section{References}

Bower, G. H. (1981). Mood and memory. American Psychologist, 36, 129-148.

Dalsky, J. D., Gohm, L. C., Noguchi, K., \& Shiomura, K. (2008). Mutual self-enhancement in Japan and the United States. Journal of Cross-Cultural Psychology, 39, 215-223.

De Beni, R., \& Moè, A. (2003). Imagery and rehearsal as study strategies for written or orally presented passages. Psychonomic Bulletin \& Review, 10(4), 975-980.

Diener, E., \& Biswas-Diener, R. (2008). Happiness: Unlocking the mysteries of psychological wealth. Malden, M.A.: Blackwell Publishing.

Emmons, R. A., \& McCollough, M. E. (2003). Counting blessings versus burdens: An experimental investigation of gratitude and subjective well-being in daily life. Journal of Personality and Social Psychology, 84(2), 377-389.

Hu, L., \& Bentler, P. M. (1999). Cut-off criteria for fit indexes in covariance structure analysis: Conventional criteria versus new alternatives. Structural Equation Modeling, 6, 1-55.

Kemp, S., Burt, C. D. B., \& Furneaux, L. (2008). A test of the peak-end rule with extended autobiographical events. Memory \& Cognition, 36(1), 132-138.

Martin, L. L., \& Clore, G. L. (2001). Theories of mood and cognition. Hillsdale: Erlbaum.

Moè, A., \& De Beni, R. (2005). Stressing the efficacy of the Loci method: Oral presentation and the subject-generation of the loci pathway with expository passages. Applied Cognitive Psychology, 19, 95-106.

Noguchi, K., Gohm, L. C., Dalsky, J. D., \& Sakaamoto, S. (2007). Cultural differences related to positive and negative valence. Asian Journal of Social Psychology, 10, 68-76.

Noguchi, K., Gohm, C. L., \& Dalsky, D. J. (2006). Cognitive tendencies of focusing on positive and negative information. Journal of Research in Personality, 40, 891-910.

Rozin, P., \& Royzman, E. B. (2001). Negativity bias, negativity dominance, and contagion. Personality and Social Psychology Review, 5(4), 296-320.

Russell, J. A. (1980). A circumplex model of affect. Journal of Personality and Social Psychology, 39, 1164-1178. 